Ann. Biol. anim. Bioch. Biophys., I973, 13 (2), I55-163.

\title{
ÉVOLUTION FETALE ET POSTNATALE DU CONTENU EN HORMONE DE CROISSANCE DE L'HYPOPHYSE OVINE
}

\author{
J. CHARRIER \\ avec la collaboration technique de Y. Carles et P. Grisolles \\ Station de Physiologie animale, I. N.R. A., \\ École nationale supérieure agronomique, \\ 9. Place Viala, \\ 34060 Montpellier Cedex
}

\begin{abstract}
RÉSUMÉ
Les hypophyses de $8 \mathrm{I}$ agneaux sont prélevées dans des conditions très normalisées, et sont dosées par le test tibia pour leur activité somatotrope. Les prélèvements sont échelonnés du stade de 105 jours de vie fœtale jusqu'à 250 jours de vie postnatale. Les caractéristiques suivantes : concentration hypophysaire, contenu hypophysaire total, quantité de $\mathrm{GH} / \mathrm{kg}$ de poids vif sont rapportées soit en fonction de l'âge, soit en fonction du poids. La concentration hypophysaire en GH reste constante après la naissance, et comprise entre 40 et $85 \mathrm{UI} / \mathrm{g}$. Le contenu hypophysaire augmente donc comme le poids de la glande. Le rapport quantité de $\mathrm{GH} / \mathbf{k g}$ poids vif marque un pic à la naissance, avec I $800 \mathrm{mUI} / \mathrm{kg}$ puis décrô̂t rapidement. Dans tous les cas, une très forte variation individuelle est enregistrée.
\end{abstract}

\section{INTRODUCTION}

Beaucoup d'auteurs ont essayé d'établir une relation entre les caractéristiques de croissance (vitesse de croissance ou poids corporel) et le contenu ou la concentration hypophysaire en hormone somatotrope (GH). Leurs techniques de dosage se rangent soit parmi les tests biologiques, seuls utilisés avant I96o, soit parmi les dosages radioimmunologiques, qui au cours des Io dernières années ont supplanté les premiers dans une très large mesure. Mais il apparaît un manque de corrélation entre les résultats obtenus avec les deux méthodes, ce qui met en question la signification biologique de l'une au moins d'entre elles (GARCIA et GESCHWIND, Ig68; RODGER et al., I969). Cependant une corrélation acceptable apparaît à la condition expresse que les hypophyses dosées soient issues d'animaux non stimulés et non manipulés (DAUGHADAY et al., I968; MüLLER et al., I972). 
Dans l'espèce humaine, les données qui concernent les teneurs hypophysaires en HGH sont peu nombreuses. Cependant les travaux de GershBERG (I957) et RICE et al. (I968) qui utilisèrent les tests biologiques, comme ceux de KAPLAN et Grumbach (I967) et GaILANI et al. (I970) qui ont employé les techniques radioimmunologiques, établissent sur des hypophyses fœtales une relation entre l'âge du fœtus et son contenu en GH, mais ne notent aucune différence entre sexes.

Chez les rongeurs, Solomon et GREEP (I958) et BowmaN (I96I) ne constatent aucune évolution postnatale du contenu en $\mathrm{GH}$ en fonction de l'âge chez le Rat, mais notent de grosses variations individuelles. BIRGE et al. (Ig67) qui trouvent également des variations considérables entre animaux de même âge et même sexe, estiment à 1'aide du dosage radioimmunologique que la concentration en GH dans l'hypophyse de rat augmente à deux périodes critiques de la vie : pendant la première semaine de vie postnatale et à la puberté.

Chez les ruminants, FRANSEn et ANDREws (I954) ne trouvent pas de différence de teneur en GH hypophysaire entre des veaux nains et normaux, alors que pour MARLOWE et Chambers (I954) il y a moins de GH chez les normaux, et pour MarLowe (I960) moins chez les nains. Pour Armstrong et Hansei, (I956) la teneur hypophysaire en $\mathrm{GH}$ diminue avec le temps chez la génisse.

Ces résultats sont donc parfois contradictoires; de plus, nous ne disposons pas de données de cet ordre chez le Mouton. C'est pourquoi nous avons entrepris cette étude, destinée à préciser, à l'aide d'un test biologique, quelle est l'évolution de la concentration et du contenu total en GH de l'hypophyse ovine et à établir si une relation existe entre la GH hypophysaire et la croissance, depuis le stade foetal jusqu'à $25^{\circ}$ jours de vie postnatale.

\section{MATÉRIEL ETT MÉTHODES}

\section{A. - Animaux}

Les hypophyses ont été prélevées sur 8I agneaux Mérinos d'Arles issus du troupeau élevé au laboratoire. Dès leur jeune âge les animaux reçoivent, en complément du lait maternel, un aliment concentré en quantité limitée (Cofna Agneau junior $\mathrm{C}_{\text {I2) }}$ ) et ont un accès libre et pe1manent au foin, ce qui leur permet de passer très progressivement de l'alimentation lactée à un aliment solide; le sevrage définitif a lieu vers un poids approximatif de $20 \mathrm{~kg}$.

Les animaux ont été abattus et leur hypophyse prélevée soit à des âges précis. correspondant à des stades déjà utilisés au laboratoire pour des travaux concernant la croissance (VÉzINHET et DAUZIER, I970 ; BENEVENT, I97I ; PRUD'HON et al., 1972), soit à des poids prédéterminés.

Le détail des stades et des effectifs figure au tableau $\mathbf{I}$.

Les fœtus, obtenus par césarienne, sont immédiatement décapités.

Chez les agneaux nous avons cherché à éviter l'influence d'un stress éventuel qui pourrait être un facteur de décharge de GH chez le Ruminant comme il l'est chez l'Homme, le Singe, le Rat (Schalch et Reichlin, I968). Bien que Purchas et al. (I97I) n'aient pas constaté de décharge par des génisses pendant leur transport à l'abattoir, nous avons été amenés à prendre certaines précautions : l'animal est assommé dans la bergerie même, à la place où il se trouve, à l'aide d'un pistolet d'abattage, puis immédiatement transporté en abattoir où il est décapité.

Pour éviter des variations dues à un éventuel rythme circadien du contenu hypophysaire en GH, déjà connu chez le Rat (MüLLER et al., I970), tous les abattages ont été effectués à 9 heures du matin.

Enfin, l'influence du jeâne semblant être sans effet sur la régulation de la sécrétion de GH chez les ruminants (Mc ATEe et Trenkle, I97I; Wallace et Bassett, I970; Wagner et al., I969; TrenkLe, 197I $a$ et $b$ ) nos animaux n'ont pas été mis à jeun avant l'abattage. 


\section{TABLEAU I}

Stades d'abattage (jour fixe ou poids fixe), poids moyen à l'abattage (entre parenthèses valeurs extrêmes) Nombre et sexe des agneaux donneurs d'hypophyse

\begin{tabular}{|c|c|c|c|c|}
\hline \multirow{2}{*}{$\begin{array}{c}\text { Age } \\
\text { (jours) }\end{array}$} & \multicolumn{2}{|c|}{ Poids (kg) } & \multicolumn{2}{|c|}{ Nombre } \\
\hline & $\delta$ & 우 & $\tilde{a}$ & q \\
\hline \multicolumn{5}{|l|}{ Fotus } \\
\hline 105 & $1,1-1,1$ & 1,1 & 2 & 1 \\
\hline 115 & $1,7-1,8$ & $1,5-1,7$ & 2 & 2 \\
\hline 140 & $3,3-3,4$ & $2,6-3,0$ & 2 & 2 \\
\hline \multicolumn{5}{|l|}{ Agneaux } \\
\hline 0 & $3,4 \quad(2,6-3,9)$ & $3,8(3,6-4,0)$ & 7 & 4 \\
\hline 10 & $5,6 \quad(5,0-5,9)$ & $5,6 \quad(4,9-6,4)$ & 4 & 4 \\
\hline $15-22$ & 7,5 & & 4 & \\
\hline 25 & $9,9(9,7-10,2)$ & $8,0 \quad(6,9-8,8)$ & 4 & 4 \\
\hline $34-37$ & 12,5 & & 4 & \\
\hline 50 & $14,9 \quad(12,4-18,4)$ & $13,6(13,0-14,8)$ & 4 & 4 \\
\hline 100 & $28,2(25,8-31,3)$ & $25,0 \quad(21,1-29,1)$ & 4 & 4 \\
\hline 150 & $31,1 \quad(29,5-32,0)$ & $26,8(23,6-30,3)$ & 4 & 7 \\
\hline 250 & $41,5 \quad(38,0-45,5)$ & $32,7(28,1-36,6)$ & 4 & 4 \\
\hline Total & & & 45 & 36 \\
\hline
\end{tabular}

Tout de suite après décapitation l'adénohypophyse seule est récupérée, pesée, broyée au Potter, et le broyat en suspension dans $1^{\prime}$ eau distillée est aussitôt congelé et conservé à $-25^{\circ} \mathrm{C}$ en attendant d'être dosé. La totalité de la manipulation dure environ 20 minutes.

\section{B. - Dosage biologique}

Nous avons utilisé le test d'accroissement en épaisseur du cartilage épiphysaire du tibia de rat hypophysectomisé (Evans et al., I943; GreensPan et al., r949), légèrement modifié par l'utilisation de femelles opérées à 32-34 jours, au lieu du 28-30 jours, et pesant 80 à Ioo g (CHARRIER, non publié).

L'hypophysectomie est pratiquée par voie transauriculaire selon la technique de FALCONI et Rossi (I964).

Notre standard de référence est de l'hormone somatotrope bovine commerciale Byla, lot 69/oI, qui, comparée au standard international de la World Health Organization, Mill Hill, London, révèle une activité biologique de $\mathrm{I}, 93 \mathrm{UI} / \mathrm{mg}$ (limites de confiance : $1,54^{-2,4 I ~ U I / m g) . ~}$

L'exploitation statistique du dosage en quatre points a été faite par analyse de variance, selon FINNEY, 1964.

\section{RÉSULTATS}

\section{A. - Croissance}

Dans l'échantillon étudié, les vitesses de croissance des mâles et des femelles ont suivi une évolution similaire (fig. I $a$ ). Le gain de poids quotidien des mâles se situe à environ $240 \mathrm{~g}$, celui des femelles à 2 ro $\mathrm{g}$, de façon constante jusqu'à Ioo jours, puis on constate un déclin progressif. 
Les poids hypophysaires (fig. I $a$ ) évoluent de façon à peu près semblable au poids corporel, avec cependant une vitesse plus faible, ce qui est en accord avec les observations de BENEVENT (I97I) qui signale, en outre, une très forte variabilité.

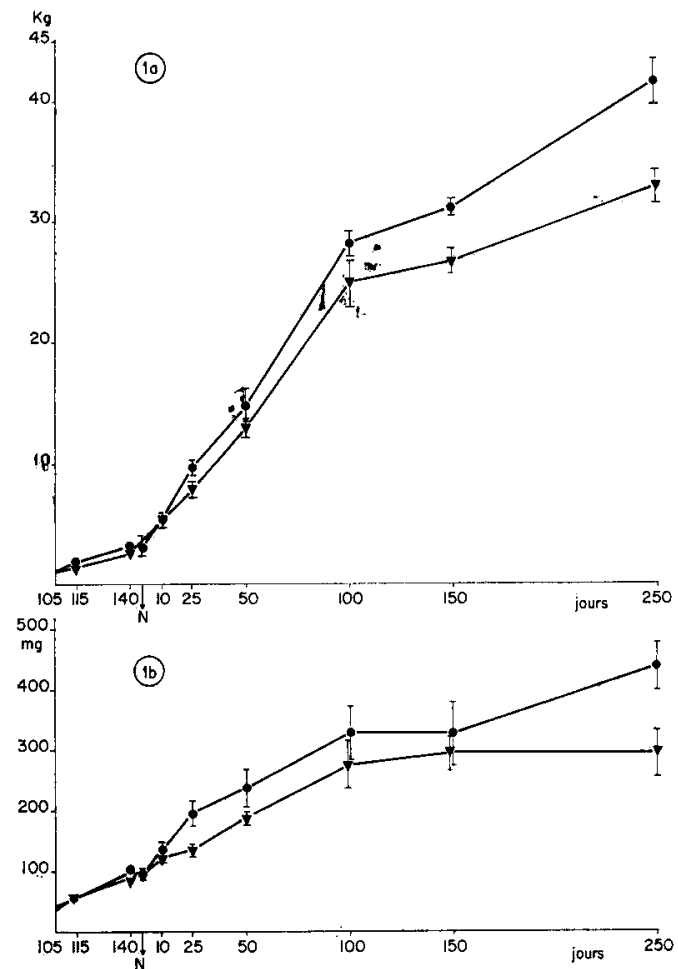

FIG. I a. - Evolution du poids corporel des agneaux donneurs d'hypophyse Chaque point : moyenne \pm erreur-type $\left(S_{m}\right)$

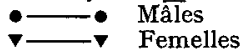

FIG. I b. - Répartition selon l'âge de l'animal du poids des préhypophyses prélevées Chaque point représente la moyenne \pm erreur-type $\left(S_{m}\right)$

$\begin{array}{ll}\bullet \longrightarrow & \text { Mâles } \\ \square & \text { Femelles }\end{array}$

\section{B. - Concentrations et contenus hypophysaires en $G H$}

Les figures 2 et 3 expriment respectivement en fonction de 1'âge et du poids, l'évolution de la concentration hypophysaire en $\mathrm{GH}(a)$, du contenu total $(b)$ et de la quantité de $\mathrm{GH}$ hypophysaire rapportée à 1'unité de poids vif $(c)$. La figure 2 représente des moyennes, alors que la figure 3 donne les valeurs individuelles. L'évolution du contenu total en GH de l'hypophyse en fonction du temps (fig. $2 b$ ) a le même profil que l'évolution du poids de 1'hypophyse (fig. I $b$ ).

La représentation du contenu total en GH en fonction du poids vif (fig. $3 b$ ) s'ajuste assez bien, avec un coefficient de corrélation $r=0,83$, à deux droites dont les pentes seraient $\mathrm{I}, 69$ et 0,55 , le changement de pente ayant lieu pour un poids d'environ $4 \mathrm{~kg}$ correspondant à la naissance. 

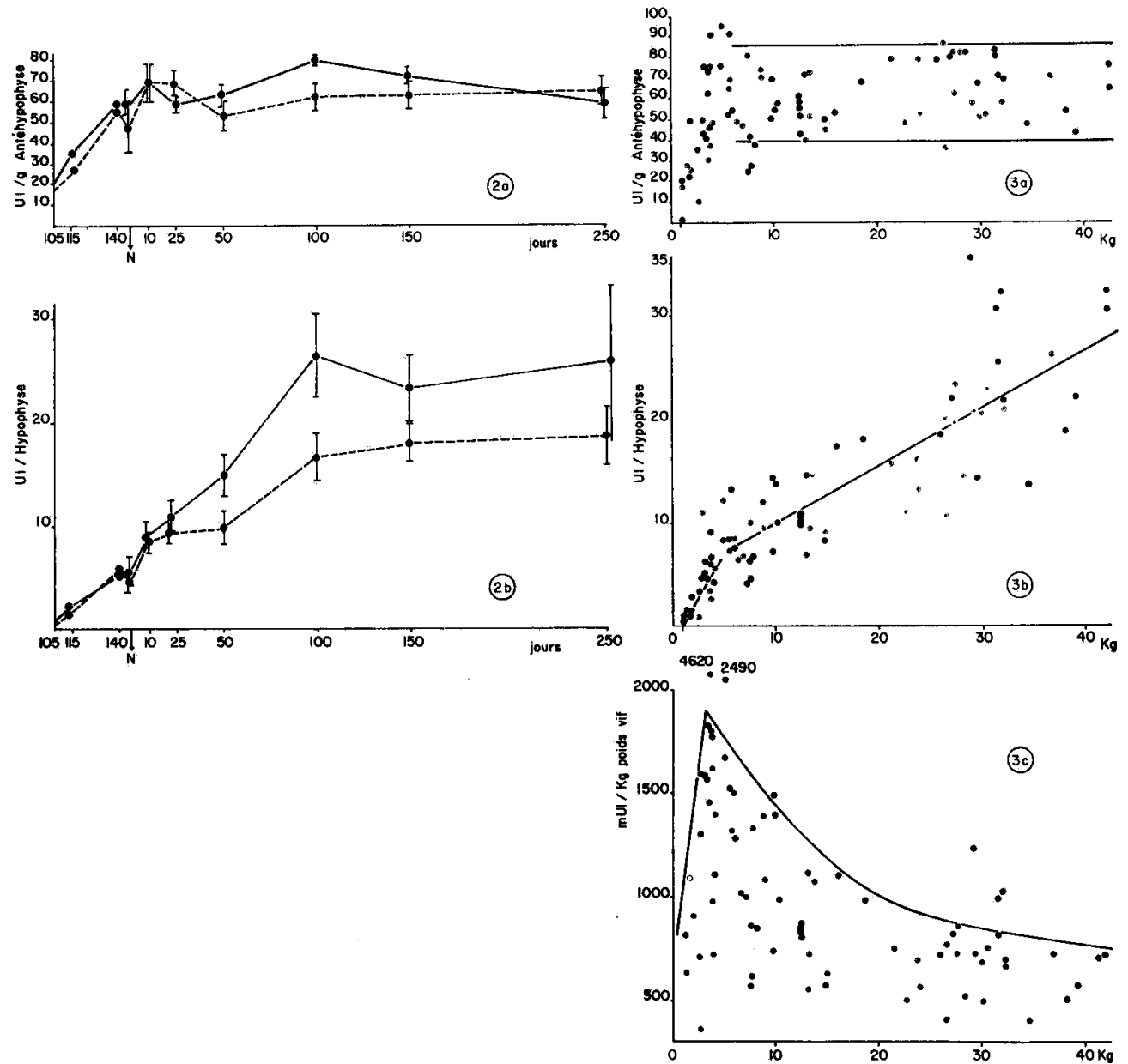

FIG. 2. - Représentation des données en fonction de l'âge

Chaque point représente une moyenne \pm erreur-type $\left(S_{m}\right)$. Pour les stades prénatals, il n'y a que 2 animaux par point et les $S_{m} n$ 'ont pas été calculées.

$$
\begin{aligned}
& \mathrm{N}=\text { naissance } \\
& \begin{array}{ll} 
& \text { Mâles } \\
\bullet-\ldots .-\bullet & \text { Femelles }
\end{array}
\end{aligned}
$$

FIG. 3. - Données individuelles en fonction du poids

\section{- Mâles}

- Femelles

Frg. a. - Évolution de la concentration préhypophysaire en hormone de croissance (GH) chez 1'Agneau, en unités internationales d'activité somatotrope par gramme de tissu glandulaire frais.

FIG. b. - Évolution du contenu total en hormone somatotrope de la préhypophyse d'agneau, en unités internationales par glande.

Fig. c. - Évolution chez l'Agneau de la quantité d'hormone somatotrope hypophysaire disponible par unité de poids corporel de l'animal. 
La concentration hypophysaire exprimée en UI par gramme de préhypophyse croît régulièrement jusqu'au $\mathrm{I}_{40^{\circ}}$ jour de gestation (fig. 2 a), qui précède la parturition de quelques jours. Après la naissance, cette concentration hypophysaire qui reste à peu près constante pendant la période étudiée, est comprise entre 40 et 85 UI par gramme d'hypophyse. C'est dans la période périnatale, soit à un poids de 3 à $4 \mathrm{~kg}$, que la variabilité individuelle est la plus forte. C'est également dans cette période que la quantité de $\mathrm{GH}$ hypophysaire par unité de poids du corps (fig. $3 \mathrm{c}$ ) atteint les valeurs les plus fortes (I $800 \mathrm{mUI} / \mathrm{kg}$ ).

\section{DISCUSSION}

STOKEs et BoDA (I968) ont détecté par immunofluorescence la présence de GH dans $1^{\prime}$ hypophyse fœtale ovine dès le $5^{0^{e}}$ jour après la fécondation, et DuBoIs (I97 I) dès le $67^{\mathrm{e}}$ jour dans $1^{\prime}$ hypophyse fotale bovine. Malgré un certain manque de sensibilité, la technique que nous avons utilisée nous a permis de doser l'activité somatotrope hypophysaire dès le $\operatorname{Io}^{\mathrm{e}}$ jour de vie fotale et de mettre en évidence une augmentation de la concentration en $\mathrm{GH}$ au cours de la croissance fœtale, ce qui est en accord avec les constatations de OXENDER et al. (I972) chez les bovins et de BAKER et al. (I956) chez la Truie.

Pendant la période postnatale nous avons mis en évidence une relative constance des valeurs moyennes des concentrations hypophysaires et une très grande variabilité des valeurs individuelles alors que ARMSTRONG et HANSEL (I956) enregistrent chez la Génisse une lente diminution de cette concentration, entre o et 80 semaines. Ces auteurs n'ont pu établir aucune corrélation entre la teneur en GH et le poids de la glande ou le poids de l'animal, et nos propres résultats retrouvent chez le Mouton cette absence de corrélation.

Le rapport $\frac{\text { quantité de GH hypophysaire }}{\text { poids du corps }}$, maximum à la naissance, diminue assez rapidement et se trouve environ trois fois plus faible chez nos ovins au bout de $25^{\circ}$ jours. La même observation avait été faite par BAKER et al. (I956) chez des Truies entre o et 300 jours, par CURL et al. (I968) chez le Veau, pour qui ce rapport est ro fois plus faible qu'à la naissance lorsque l'animal atteint le poids de $200 \mathrm{~kg}$, et chez le Porc par BAIRD et al. (I952). Ceux-ci avaient d'ailleurs émis l'hypothèse que l'arrêt de croissance pourrait être dû au passage de ce rapport au-dessous d'une certaine valeur seuil.

Il est quelque peu surprenant de trouver la plus forte valeur pour ce rapport dans la période périnatale, alors que justement dans les quelques jours qui précèdent la naissance 1'évolution ponđérale semble marquer un palier. Parallèlement au niveau plasmatique BASSETT et AlEXANDER (I970), WALLACE et BASSETT (I970) et BASSETT et al. (I970), trouvent dans le plasma de fœtus ovin en fin de gestation une concentration très élevée en GH, qui chute dans les heures qui suivent la mise bas. Chez les bovins, PURChas et al. (I970) font les mêmes observations.

Ainsi, le rôle joué par la fonction hypophysaire au stade fœtal et pendant le jeune âge des mammifères, est encore très obscur : des fœtus de lapin (JosT, I947) ou de rat (Jost, I95I) décapités, ont une croissance quasi-normale jusqu'à leur 
mise bas. Des enfants nouveau-nés souffrant d'une aplasie hypophysaire, et qui meurent dès leur naissance, ont pourtant un poids normal (BREWER, I957). Néanmoins certains résultats indiquent que la croissance prénatale peut dépendre dans une certaine mesure d'une sécrétion d'origine fotale. Pour HEGGESTAD et WELIS (I965), la croissance fœtale des rats dépendrait pour 20 p. Ioo de 1'hypophyse dans les derniers temps de vie intrautérine. Chez les ovins, l'hypophysectomie du fœtus provoque un retard de développement somatique d'autant plus marqué que l'opération a lieu tôt au cours des 50 derniers jours de gestation (LIGGINS et KENNEDY, I968). Par ailleurs, après la naissance, les sécrétions hypophysaires semblent n'être pas indispensables au développement. L'hypophysectomie, à 1'âge de 25 jours, stoppe la croissance de nos agneaux (VEZINHET, I968 $a$ ) et même dès 1'âge de ro jours (VÉZINHET, communication personnelle), alors qu'elle ne devient efficace chez le Rat qu'à la $4^{\text {e }}$ semaine d'âge (WAI,KER et al., I950), et pas avant Ioo jours chez le Lapin (VÉzINHET, r968 b).

Notre but, rappelons-le, était de rechercher s'il existait ou non une corrélation entre 1'hormone de croissance hypophysaire et la croissance chez le Mouton. Chez les bovins, HaFs et al. (I97I) n'avaient pu réussir par la technique radioimmunologique à établir une corrélation entre la croissance corporelle et les différentes caractéristiques hypophysaires et plasmatiques de la GH (quantité totale et concentration). Nous ne pouvons qu'arriver aux mêmes conclusions dans les limites de validité et de sensibilité de notre dosage, en ce qui concerne la GH hypophysaire des ovins.

Reçu pour publication en février 1973.

\section{REMERCIEMENTS}

Ce travail a été réalisé en partie grâce au contrat D. G. R. S. T. "Biologie de la Reproduction et du Développement - Développement postnatal ", no 7 I 73 I29.

Nous tenons à remercier ici les docteurs FALCONI et Rossi, Instituto Vister, Casatenovo (Como), Italie, pour le don généreux de leur propre aiguille à hypophysectomiser les rats. Nous remercions également l'Organisation Mondiale de la Santé (W.H. O. Mill Hill, London) pour la fourniture du standard international d'hormone somatotrope. Notre reconnaissance va au docteur Stoliaroff (Laboratoire Byla, 9I - Massy, France) et aux docteurs Pecile et Müller (Instituto di Farmacologia, Milano, Italie) pour nous avoir initiés à la technique du dosage biologique et du test tibia.

\section{SUMMARY}

FOETAL AND POST NATAL, PROGRESS OF GROWTH

HORMONE CONTENT IN THE SHEEP PITUITARY

Pituitary glands from 8I lambs are obtained in very standardized conditions, and their growth hormone content assayed by the tibia test. Glandular samples are taken from to5 days of foetal life to 250 days after birth. The following features — pituitary level, total pituitary content and $\mathrm{GH}$ content/live weight ratio - are reported in relation to age and weight. After birth, GH pituitary concentration remains constant at 40 to $85 \mathrm{IU} / \mathrm{g}$. The pituitary GH content/ live weight ratio shows a peak of $\mathrm{I} 800 \mathrm{mIU} / \mathrm{kg}$ at birth, then rapidly decreases. At each stage, large individual variation is noticed. 


\section{RÉFÉRENCES BIBLIOGRAPHIQUES}

Armstrong D. T., Hansel W., I956. The effect of age and plane of nutrition on growth hormone and thyrotropic hormone content of pituitary glands of Holstein heifers. J. Anim. Sci., 15, 640-649.

Batrd D. M., Nalbandov A. V., Norton H. W., I952. Some physiological causes of genetically different rates of growth in swine. J. Anim. Sci., 11, 292.

Baker B., Hollandbeck R., Norton H. W., Nalbandov A. V., 1956. Growth hormone content of swine pituitaries in relation to growth rate and age. J. Anim. Sci., 15, 407-4I7.

Bassett J. M., Alexander G., I975. Insulin, growth hormone and corticosteroïds in neonatal lambs. Biol. Neonate., 17, I12-125.

Bassett J. M., Thorburn G. D., Wallace A. L. C., 1970. The plasma growth hormone concentration of the foetal lamb. J. Endocr., 48, 25I-263.

Benevent M., rg7r. Croissance relative pondérale postnatale, dans les deux sexes, des principaux tissus et organes de l'Agneau Mérinos d'Arles. Ann. Biol. anim. Bioch. Biophys., 11, 5-39.

Birge C. A., Peake G. T., Mariz I. K., Daughaday W. H., I967. Radio-immunoassayable growth hormone in the rat pituitary gland : effects of age, sex and hormonal state. Endocrinology, 81, 195-204.

Bowman R. H., I96r. Growth hormone activity of the anterior pituitary lobe of the male rat at various ages. Nature, 192, 976-977.

Brewer D. B., I957. Congenital absence of the pituitary gland and its consequences. J. Path. Bact., 73, $59-67$.

Curl S. E., Fennell M. A., Zinn D. W., Albin R. C., ig68. Growth and development of the bovine as related to certain endocrine factors. J. Anim. Sci., 27, roII.

Daughaday W. H., Peake G. T., Birge C. A., Mariz I. K., I968. The influence of endocrine factors on the concentration of growth hormone in rat pituitary. In : Growth hormone, p. 238. Editors : A. Pecile and E. E. Müller, Excerpta Medica, Amsterdam.

Dubors M., I97r. Mise en évidence par immunofluorescence des cellules somatotropes et des cellules à prolactine dans l'hypophyse foetale des bovins. C. R. Acad. Sci., 272, 433-435.

Fransen J. M., Andrews F. N., x954. The physiology of dwarfism in beef cattle. J. Amim. Sci., 13, I020.

Gailani S. D., Nussbaum A., Mc Dougall W. J., Mc Limans W. F., r97o. Studies on hormone production by human fetal pituitary cell cultures. Proc. Soc. exp. Biol. Med., 134, 27-32.

Garcia J. F., GeSchwind I. I., I968. Investigation of growth hormone secretion in selected mammalian species. In: Growth hormone, p. 267. Editors: A. Pecile and E. E. Müller, Excerpta Medica, Amsterdam

GershberG H., 1957. Growth hormone content and metabolic actions of human pituitary glands. Endocrinology, 61, 160 .

Hafs H. D., Purchas R. W., Pearson A. M., 1971. A review : relationships of some hormones to growth and carcass quality of ruminants. J. Anim. Sci., 33, 64-7I.

Heggestad C. B., Wells L. J., I965. Experiments on the contribution of somatotrophin to prenatal growth in the rat. Acta Anat., 60, 348-36r.

Jost A., r947. Expériences de décapitation de l'embryon de lapin. C. R. Acad. Sci. Paris, 225, 322-324

Jost A., r95I. La physiologie de l'hypophyse fotale. Biol. $M e \hat{e}$, , 40, 205-229.

KaPLAN S. L., GRUMBach M. M., I967. Growth hormone secretion in the human fetus and in anencephaly. Ith Intern. Symp. on growth Hormone, Milan. Excerpta Medica Serv., 142, $5 \mathrm{I}$.

LigGins G. C., KENNEDY P. C., I968. Effects of electrocoagulation of the foetal lamb hypophysis on growth and development. J. Endocr. 40, 37I-381.

Mc Atee J. W., TRENkle A., I97I. Effect of feeding, fasting and infusion of energy substrates on plasma growth hormone levels in cattle. J. Anim. Sci., 33, 6r2-6r6.

MARLowe T. J., r960. A comparison of the growth hormone content of the pituitary glands from dwarf and normal beef calves, J. Anim. Sci., 19, 810-819.

Marlowe T. J., Chambers D., r954. Some endocrine aspects of dwarfism in beef cattle. J. Anim. Sci., 13, $96 \mathrm{I}$.

Müller E. E., Giustina G., Miedico D., Cocchi D., Pecile A., I972. Analogous pattern of bioassayable and radioimmunoassayable growth hormone in some experimental conditions of rat and mouse. In Growth and growth hormone, p. 283. Editors A. Pecile and E. E. Müller. Excerpta Medica, Amsterdam.

Müller E. E., Giustina G., Miedico D., Pecile A., Cocchi D., Wang King F., I97o. Circadian pattern of bio-assayable and radioimmunoassayable growth hormone in the pituitary of female rats. Proc. Soc. exp. Biol. Med., 135, 934-939.

Prud'hon M., Reyne Y., Garambois X., I972. Estimation de la composition corporelle d'agneaux Mérinos d'Arles abattus à des stades de croissance compris entre la naissance et un an. Ann. Zootech., 21, 299-309. 
Purchas R. W., Mc Millan K. L., Hafs H. D., i97o. Pituitary and plasma growth hormone levels in bulls from birth to one year of age. J. Anim. Sci., 31, 358 .

Purchas R. W., Pearson A. M., Hafs H. D., Tucker H. A., I97I. Some endocrine influences on the growth and carcass quality of Holstein heifers. J. Anim. Sci., 33, 836.842.

OXender W. D., Conwey E. M., HAFS H. D., I972. Bovine fetal pituitary concentration and in vitro synthesis of prolactin, growth hormone and luteinizing hormone. Proc. Soc. exp. Biol. Med., 139, I0r7-I02x.

Rice B. F, Ponthier R., Sternberg W., Ig68. Luteinizing hormone and growth hormone activity of the human fetal pituitary. J. Clin. Endocr. Metab., 28, 1071.

Rodger N.W., Beck J.C., Burgus R., Guillemin R., I969. Variability of response in the bioassay for a hypothalamic somatotrophin releasing factor based on rat pituitary growth hormone content. Endocrinology., 84, 1373-1383.

Schalch D. S., Reichlin S., I968. Stress and growth hormone release. In : Pecile A., Müller E. E. Growth hormone, 21 I-225. Excerpta Medica, Amsterdam.

Solomon J., Greep R. O., I959. The growth hormone content of several vertebrate pituitaries Endocrinology, 65, 334-336.

STokes H., BoDA J. M., I968. Immunofluorescent localization of growth hormone and prolactin in the adenohypophysis of fetal sheep. Endocrinology, 83, 1362-1366.

TRENkLe A., I97I a. Influence of blood glucose levels on growth hormone secretion in sheep. Proc. Soc. exp. Biol. Med., 136, $5 \mathrm{I}-55$.

Trenkle A., I97I $b$. Effect of diet upon levels of plasma growth hormone in sheep. J. Anim. Sci., 32, III-II4.

Vézinhet A., I 668 a. Effet de l'hypophysectomie sur la croissance pondérale de l'Agneau. $C$. $R$. Acad. Sci. Paris, 266, 388-39o.

Vézinhet A., rg68 b. Effet de l'hypophysectomie sur la croissance pondérale du Lapin. C. R, Acad. Sci. Paris, 266, 2348-235I.

VÉzinhet A., DAuzier L., I97o. Influence de traitements à l'hormone somatotrope bovine sur la croissance pondérale d'agneaux normaux ou hypophysectomisés. Ann. Biol. anim. Bioch. Biophys., 10, 5-I3.

Wagner J. F., Veenhuizen E. I., Root M. A., 1970. Growth hormone in the lamb. J. Anim. Sci., 31, 232-233.

Wallace A. L. C., Bassett J. M., I970. Plasma growth hormone concentrations sheep measured by radioimmunoassay. J. Endocr, 47, 21-36. 